\title{
Case Series
}

Open Access, Volume 2

\section{Pulmonary mucormycosis: A rare entity. Cases with bronchial necrosis and bronchomediastinal fistula}

\author{
Yukti Sharma; Sushil Kumar Chumber; Madhu Kanodia
}

St Stephens Hospital, Tis Hazari, Delhi 110054, India.

\author{
*Corresponding Author: Yukti Sharma \\ Consultant, St Stephens Hospital, Tis Hazari, Delhi \\ 110054, India. \\ Email: dryukti2006@yahoo.com
}

Received: Sep 30, 2021

Accepted: Nov 19, 2021

Published: Nov 26, 2021

Archived: www.jcimcr.org

Copyright: (C) Sharma Y (2021).

DOI: www.doi.org/10.52768/2766-7820/1428

\section{Abstract}

Mucormycosis is a rare fulminant fungal infection associated with high morbidity and mortality rates. It is usually associated with diabetes mellitus, hematologic malignancy, renal failure, or immunosuppression. Of the various clinical manifestations pulmonary involvement is the second most common presentation after rhino orbital cerebral disease. There is a dearth of data regarding the reports of pulmonary mucormycosis from India. We present 2 cases of pulmonary mucormycosis diagnosed as per EORTC guidelines as proven and possible mucormycosis. One case presented with DKA. The other patient was diagnosed with AAV. Both patients were treated with liposomal amphotericin $B$ and surgical resection. Both expired, presumably as a result of disseminated mucormycosis.

Keywords: ketoacidosis; mucormycosis; bronchopleural fistula.
Case I

A 62 year male, tea seller by occupation developed low grade fever, cough with expectoration of 12 days duration and breathlessness at rest. He complained of increased frequency of micturation, constipation and decreased appetite. He was recently diagnosed with DM 2 and hypertension.

On admission, the patient was conscious and oriented. Blood pressure was $140 / 80 \mathrm{mmHg}$, pulse rate was $120 / \mathrm{min}$, regular, low volume. His vital signs were: Temperature $97.4^{\circ} \mathrm{F}$, respiratory rate $30 / \mathrm{min}$, using accessory muscle of respiration with oxygen saturation $94 \%$ on low flow oxygen. Dyspnoea and pedal edema were present. The patient appeared ill but the physical examination was otherwise unremarkable. He was admitted for severe respiratory distress and diabetic ketoacidosis.

Laboratory results revealed a raised white blood cell count of $30,000 /$ cumm with neutrophilic predominance, blood glucose $454 \mathrm{mg} / \mathrm{dL}$, creatinine clearance $0.84 \mathrm{~mL} / \mathrm{min} / 1.73 \mathrm{~m}^{2}$, and electrolyte imbalance. HbA1C was 15.1, and DKA management was started. Patient was continued on BIIPAP support.

Radiology findings on day 2 showed significant opacity of entire left hemithorax, indicative of left pleural effusion with few scattered nodular patches in right lower zone S/O infection. $X$ ray findings next day showed reduction in left pleural effusion, with evidence of minimal expansion of left lung right lung mid zone showed signs of breakdown. On consecutive day bilateral CP angle blunted seen. CECT thorax findings showed left hydropnemothorax with located pnemothorax with underlying left lung subsegmental collapse and parenchymal destruction with diffuse emphysematous changes. Focal consolidation with cystic changes in right upper lobe with few nodes in left lower lobe and pericardial effusion with pericardial thickening? Infective etiology was suspected.

Lung biopsy yielded necrotic material or lung tissue containing nonseptate hyphae with rare, obtuse-angle branching consistent with Zygomycosis. No malignant cells reported. Liposomal amphotericin B was initiated at $10 \mathrm{mg} / \mathrm{kg} / \mathrm{d}$ from day 3 of hospitalization. There was concern that pneumonia might have precipitated DKA. Patient received Piperacin tazobactum (21days), clarithromycin (21days), Levofloxacin $50 \mathrm{mg}$ OD for 14 days, Liposomal Amphotericn B 4.125 G cumulative dose given over 13 days and fluconazole for 21 days. Once there was improvement in respiratory distress, patient was transferred to the general ward for further management. A chest X-ray revealed a left sided white out. Albumin infusions were given daily for hypoproteinaemia and resistant scrotal and pedal edema. Oxygen 
Citation: Sharma Y, Chumber SK, Kanodia M. Pulmonary mucormycosis: A rare entity. Cases with bronchial necrosis and bronchomediastinal fistula. J Clin Images Med Case Rep. 2021; 2(6): 1428.

therapy was continued. Subsequent $X$ ray showed resolution of pneumonia. $X$ ray one week later showed presence of pneumothorax and destruction of left lung. CECT chest confirmed the findings. Patient was planned for chest tube insertion. The patient had a prolonged hospital course (21 days) complicated by progressive hypoxemia, acute kidney injury, and toxic metabolic encephalopathy. Isolated pulmonary mucormycosis is extremely rare and carries a poorer prognosis than rhino-orbitocerebral disease.

\section{Case II}

A 54 year female, k/c/o DM and recently diagnosed hypertension was referred to the hospital in September 2018, for an inadequately responding fever and congestion in the right lung. Patient gave a history of fever of 3 months duration, rashes, and continuous headache. She was diagnosed with pulmonary Kochs elsewhere and ATT was started from which she developed side effects following which medication was discontinued after 2 doses.

O/E patient was conscious, oriented, afebrile, pallor was present. Bilateral axillary lymph nodes were enlarged. BP was $140 / 88 \mathrm{mmHg}$, pulse rate was $110 / \mathrm{min}$. General examination was unremarkable. Chest examination revealed harsh vescicular bronchial breathing, bilateral with no additional sounds. No neurological deficit but right wrist drop was seen.

HRCT thorax done showed ground glass opacities with subsegmental consolidation, septal thickening in right lung and fibrosis with tractional bronchiectasis noted in the left lower lobe. NCCT thorax done in the following week showed right sided gross hydropneumothorax with collapse of underlying lung along with a suspicious communication with right pleural cavity. (Figure) NCCT thorax done in following week showed mild hydropneumothorax in basal region of right side, multifocal ground glass opacities and consolidation with collapse consolidation right lower lobe s/o infective etiology.

Patient was treated with linezolid for 2 weeks, but fever persisted. USG showed minimal right kidney hydronephrosis. The patient was evaluated for bronchomediastinal fistula and recommended proceeding with further bronchoscopic intervention. Bronchoscopy was done; BAL fluid was negative for AFB. Gram s stain reported Gram positive cocci whereas Gene expert for AFB was negative. On culture, Escherichia coli was isolated, sensitive only to cotrimoxazole. $\mathrm{KOH}$ of $\mathrm{BAL}$ sample reported broad, ribbon-like aseptate hyphae. Fungal culture isolated Leichthemia spp. Histopathology reported right nasal cavity and paranasal sinus consistent with invasive fungal infection. Other investigations showed raised TLC, elevated RA factor, positive ANA FT, DCT ++, anti MPO levels elevated, ANCA positive vasculitis. A diagnosis of medium to small vessel vasculitis with RPGN? Wegener's Granlomatosis mononeuritis multiplex was made. In view of proteinuria, hematuria and rising creatinine levels and alveolar haemorrhages, renal biopsy was advised and plasmapheresis was planned. Serum protein electrophoresis was s/o chronic inflammation and no $\mathrm{M}$ spike seen. Patient was shifted to ICU in view of CECT chest s/o alveolar haemorrhage and planned for repeat bronchoscopy. The patient had a prolonged hospital course of one month duration complicated by progressive pneumothorax, haemoptysis and respiratory distress. CT chest again showed right sided gross pneumothorax. Patient had falling $\mathrm{BP}$, respiratory failure and tachypnoea for which she was intubated and put on ventilator. She went into renal failure followed by cardiac arrest. CPR was done but patient could not be revived.

\section{Methods}

According to the criteria put forth by the European Organization for the Research and Treatment of Cancer/Mycoses Study Group (EORTC/MSG) for Invasive Fungal Disease (IFD), [7] patients with mycological and histo-pathological evidence of mucormycosis in tissue biopsy (including observation of aseptate hyphae branching at wide angle and ribbon-like hyphae

\section{Definitions}

Proven PM was defined by histological evidence of tissue invasion consisting of non-septate, right-angle branching fungi plus recovery of Mucorale species by culture of specimens from pulmonary tissue or positivity for immunohistochemical staining with anti-Rhizopus arrhizus monoclonal antibody. Probable PM was defined as the presence of host factors together with one or more clinical indications, such as dense, well-circumscribed lesions with or without a halo sign, and an air-crescent sign or cavity on CT, and mycological evidence of Zygomycetes

in sputum or Bronchoalveolar Lavage (BAL) fluid culture, as defined by the revised criteria of the European Organization for Research and Treatment of Cancer/Mycosis Study Group [7].

The diagnosis of mucormycosis was made initially by screening for aseptate hyphae by $10 \% \mathrm{KOH}$, which was later confirmed by mycology culture. Histopathology examination of biopsy samples reported extensive necrosis and infiltration by broad, aseptate fungal hyphae branching at right angle. Treatment was promptly started irrespective of proven/ probable status of mucormycosis.

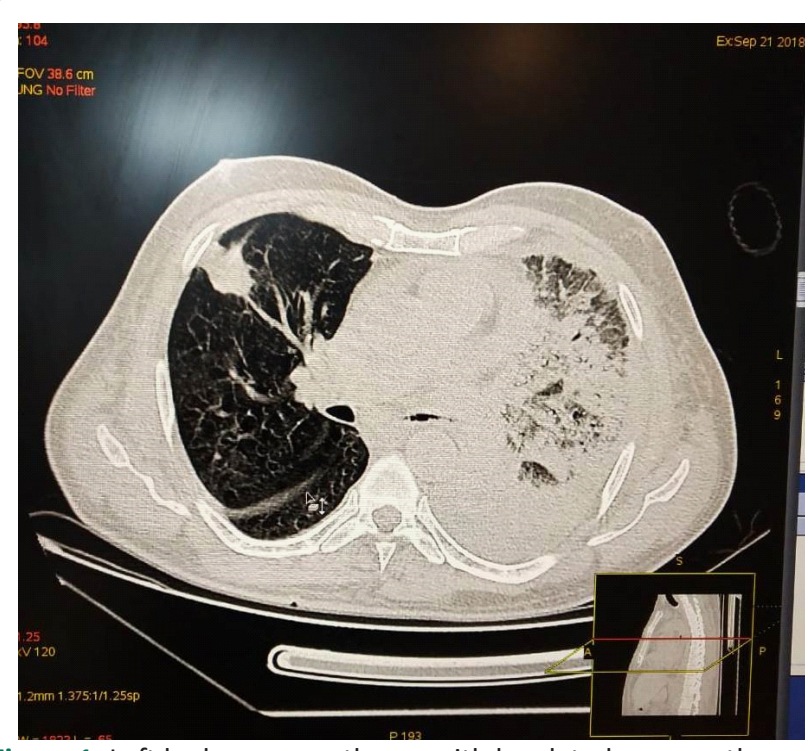

Figure 1: Left hydropneumothorax with loculated pneumothorax with underlying left lung subsegmental collapse, parenchymal destruction with diffuse emphysematous changes. Few nodules in right upper lobe. 


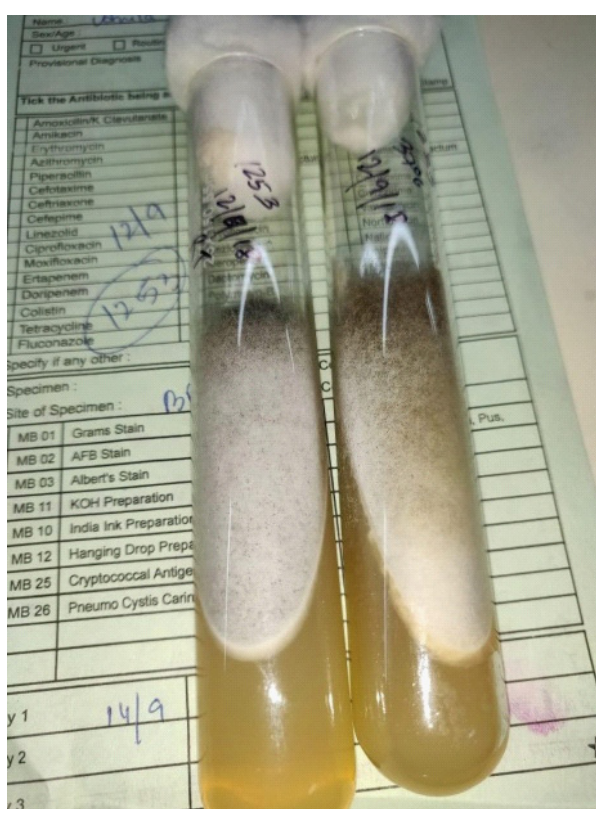

Figure 2: Rhizopus spp. Isolated at room temperature and $37^{\circ} \mathrm{C}$.

\section{Discussion}

We present two cases of pulmonary mucormycosis. Case 1 presented in diabetic ketoacidosis and case 2 had DM and later diagnosed with AAV. Clinically diagnosed with Bronchopleural fistula, bronchoscopy was advised following which liposomal amphotericin B was started for both the cases.

Mortality rates of pulmonary mucormycosis range from 50 to $80 \%$ [1]. In a large review of 929 cases of zygomycosis, an overall mortality of $44 \%$ was reported in diabetics with mucormycosis and $76 \%$ mortality for pulmonary mucormycosis in patients with other predisposing factors. Pulmonary mucormycosis occurs due to the inhalation of fungal spores with rapid progression to pneumonia or pneumonia like illness. Factors like ketoacidosis, uncontrolled diabetes mellitus, renal failure, solid tumors, immunosuppressive therapy, and solid organ transplantation have been suggested for predisposing mucormycosis [1]. Clinical presentations have a varied spectrum ranging from nonspecific symptoms like fever, dyspnea, cough, and chest pain to rare complications like progressive subcutaneous emphysema, Pancoast syndrome, Horner's syndrome, and bronchial perforation/ fistula [3].

Case 1 was admitted with bilateral pneumonia and predisposed due to DM with DKA. The second case was challenging. She was a known diabetic, recently diagnosed hypertension, paralysed and bed-ridden for last 10 years. There was pulmonary, renal and other systemic involvement. A diagnosis of RPGN (mononeuritis multiplex? ANCA associated vasculitis) was made. The antineutrophil cytoplasmic antibody (ANCA) associated vasculitides are a collection of relatively rare autoimmune diseases of unknown cause, characterized by inflammatory cell infiltration causing necrosis of blood vessels [4]. The diagnosis is based on the presence of clinical manifestations with characteristic histopathological findings and the presence of MPO-ANCA or PR3-ANCA $[4,5]$.

Renal injury has been closely associated with a poor outcome in AAV patients, as was seen in this case also [2,4,5]. Most common pathogens causing infections in patients with AAV are bacteria, followed by fungi and viruses [2]. Fungi such as Candida albicans, C. tropicalis, Aspergillus fumigates and A.flavus were reported in AAV patients by yang et al. $[2,4]$ Inflammation, renal injury, lung involvement, and immunosuppressive therapy are certain risk factors that contribute to infections by opportunistic pathogens. AAV along with pulmonary mucormycosis might have led to the grave consequences despite prompt diagnosis and aggressive treatment $[4,6]$.

Direct specimen examination with culture \&/ histopathology remains the cornerstone for diagnosis. Case no 1 is proven mucormycosis whereas case 2 falls under probable mucormycosis as per the criteria put forth by the European Organization for the Research and Treatment of Cancer/Mycoses Study Group (EORTC/MSG) for Invasive Fungal Disease (IFD) [5].

On hospital day 3, acid fast bacilli smear results were negative and Rhizopus was isolated both in bronchiolar alveolar lavage culture and necrotic tissue biopsy of case 1 . Leicthemia was isolated from BAL cultures of case no. 2.

Pulmonary haemorrhages were seen in both cases with further complications like pleural effusion and bronchopleural fistula. Pulmonary mucormycosis is an antemortem finding in $50 \%$ of the cases [1]. Rhizopus spp. Is the most commonly isolated organism.

The radiological manifestations of PM have a wide array of presentations varying from consolidation, cavitation, reversed halo sign, multiple nodules /masses, bronchopleural fistulae, lymphadenopathy and pleural effusion. High resolution CT scan (HRCT) helps in determining the evidence and extent of infection [8]. HRCT of both cases showed evidence of ground glass opacities with dense consolidations and septal thickenings, typical radiology findings of pulmonary mucormycosis. This halo presumably represents hemorrhage, and its large size likely indicates that mucormycosis results in greater pulmonary hemorrhage than other angioinvasive infections, such as Aspergillus infections, that typically have only a small halo around the nodules or even a reverse halo sign. In addition, the presence of pleural effusions and more than 10 nodules is more suggestive of pulmonary mucormycosis whereas aspergillosis is associated with small nodules less than 10 in number.

Pleural effusion with emphysema were additional findings seen in case no 1 . Fistulas are also an uncommon finding and bronchopleural fistulas were diagnosed in both the patients. ( ) Pleural effusion although uncommon in PM, was clinically as well as radiologically confirmed in case 1.

Identification of the hyphae in tissue by using endobronchial or percutaneous sampling can allow confirmation of diagnosis of mucormycosis [8].

Differentiating Mucormycosis and pulmonary aspergillosis is important since antifungal therapy varies significantly. Firstly, Mucormycosis is treated with amphotericin whereas aspergillosis responds to voriconazole. Secondly, voriconazole prophylaxis has significant association with predisposition to mucormycosis [10]. Clinically, it is difficult to differentiate pulmonary mucormycosis and aspergillosis. Unlike aspergillosis, pulmonary mucormycosis has a prognosis and outcome that have not significantly improved over the last decade, mainly due to the difficulty in forming an early diagnosis and the limited activity of current antifungal agents against Mucorales [9].

Despite being nephrotoxic, amphotericin B (1-1.5 mg/kg/ day) still remains the gold-standard therapy against mucormycosis. The recommended therapy for pulmonary mucormyco- 
sis is early surgical debridement along with early amphotericin B therapy ( $<6 \mathrm{~d}$ after diagnosis) [14]. Drugs like Posaconazole alone or in combination with amphotericin or itraconazole have been showing positive results as both these agents seem to have in vitro activity against mucormycosis pathogens. Voriconazole is ineffective against mucormycosis [10]. Mucormycosis management requires a 3-pronged approach inclusive of medical treatment, debridement of necrotic tissue and correction of underying conditions and prompt administration of liposomal Amphotericin B [11,12].

\section{Conclusion}

Despite aggressive treatment, pulmonary mucormycosis carries a high mortality rate as high as (76\%) which increases to $95 \%$ with dissemination, as compared to ROCM [13]. After an initial apparent improvement; there may be further deterioration of the symptoms, in some patients, which may point towards the active ongoing fungal infection. We agree with the guidelines that early diagnosis and aggressive treatment might reduce the mortality associated with this devastating fungal infection. Microbiological screening as well as confirmatory tests, along with histopathology reports provides an early diagnosis. Radiology reports might be non-specific. Pulmonary mucormycosis is a rare entity, thereby making the diagnosis challenging.

\section{Statements \& declarations}

\section{Authorship Policy:}

- Conceived of or designed study- Dr Yukti Sharma, Dr SK Chumber

- Performed research- Dr Yukti Sharma, Dr Madhu Kanodia

- Analyzed data- Dr Yukti Sharma, Dr SK Chumber

- Contributed new methods or models- Dr SK Chumber, Dr Madhu Kanodia

- Wrote the paper- Dr Yukti Sharma

Acknowledgements: We acknowledge the hardwork and technical assistance provided by Ms Masha Goel, technical staff at St Stephen's Hospital.

Funding: No outside funding provided.

Declarations (ethics): Approved by Ethics Committee St Stephen's Hospital, no. SSHEC/R0181.

\section{References}

1. Roden MM, Zaoutis TE, Buchanan WL, et al. Epidemiology and outcome of zygomycosis: a review of 929 reported cases, Clinical Infectious Diseases. 2005; 41: 634-653.

2. Risk factors for infectious complications of ANCA-associated vasculitis: A cohort study Liu Yang, Honglang Xie, Zhengzhao Liu, Yinghua Chen, Jinquan Wang, Haitao Zhang, Yongchun Ge* and Weixin Hu*Yang et al. BMC Nephrology. 2018; 19: 138.

3. Mekki OS, Hassan A, Falemban A, Alkotani N, Alsharif S, et al. Pulmonary Mucormycosis: A Case Report of a Rare Infection with Potential Diagnostic Problems Case Reports in Pathology. 2020: 5845394.

4. Wall N, Harper L. Complications of long-term therapy for ANCAassociated systemic vasculitis. Nat Rev Nephrol. 2012; 8: 52332.

5. Grajales C, Pineda J. Pathophysiological Relationship between Infections and Systemic Vasculitis Autoimmune Diseases. 2015: 286783.

6. Revised Definitions of Invasive Fungal Disease from the European Organization for Research and Treatment of Cancer/ Invasive Clin Infect Dis. 2008; 46: 1813-1821.

7. Petrikkos G, Skiada A, Lortholary O, Roilides E, Walsh T, Kontoyiannis D. Epidemiology and Clinical Manifestations of Mucormycosis Clinical Infectious Diseases. 2012; 54: S23-34.

8. Agrawal R, Yeldandi A, Savas $H$, Parekh N, Lombardi P, et al. RadioGraphics. Pulmonary Mucormycosis: Risk Factors, Radiologic Findings, and Pathologic Correlation. 40.

9. Spellberg B, Edwards J Jr, Ibrahim A. Novel perspectives on mucormycosis: Pathophysiology, presentation, and management. Clin Microbiol Rev. 2005; 18: 556-569.

10. Russell E. Lewis, Guangling Liao, Weiqun Wang, Randall A. Prince \& Dimitrios P. Kontoyiannis. Voriconazole pre-exposure selects for breakthrough mucormycosis in a mixed model of Aspergillusfumigatus-Rhizopusoryzae pulmonary infection, Virulence. 2011; 2: 348-355.

11. Jeong $\mathrm{W}$, et al. The epidemiology and clinical manifestations of mucormycosis: A systematic review and metaanalysis of case reports, Clinical Microbiology and Infection. 2018.

12 Uña JR Donado, Díaz Hellín V, López Encuentra A and Echave Sustaeta JM: Persistent cavitations in pulmonary mucormycosis after apparently successful amphotericin B. Eur J Cardiothorac Surg. 2002; 21: 940942.

13. Wang $X i$, guo $L$, xue $S$ and chen $Y$. Pulmonary mucormycosis: $A$ case report and review of the literature. Oncology letters. 2016; 11: 3049-3053.

14. Spellberg B, Ibrahim AS. Recent advances in the treatment of mucormycosis. Curr Infect Dis Rep. 2010; 12: 423-429. 